\title{
Multisection of series
}

\author{
A. G. Shannon \\ Warrane College, the University of New South Wales \\ Kensington, NSW 2033, Australia \\ e-mails: t . shannon@warrane . unsw . edu . au, \\ tshannon38@gmail.com
}

Received: 8 December 2018

Revised: 27 July 2019

Accepted: 29 July 2019

Abstract: This paper extends some ideas of Leonard Carlitz with ideas from John Riordan and validated against results of Alwyn Horadam. They involve breaking up series and considering multiple sums of ordinary and generalized binomial coefficients.

Keywords: Generating function, Binomial coefficient, Primitive root, Lucas numbers, Kronecker delta, Binet formulas, $q$-series.

2010 Mathematics Subject Classification: 11B65, $11 \mathrm{~B} 39$.

\section{Introduction}

This is an attempt to extend Carlitz' multisection techniques to generalized binomial coefficients [1]. Carlitz has utilized multivariable generating functions to treat the sums of products of what Riordan [2] calls the cycles of binomial coefficients. These cycles have the form

$$
\left(\begin{array}{c}
i+i_{1} \\
i_{1}
\end{array}\right)\left(\begin{array}{c}
i_{1}+i_{2} \\
i_{2}
\end{array}\right) \ldots\left(\begin{array}{c}
i_{n-1}+i_{n} \\
i_{n}
\end{array}\right)\left(\begin{array}{c}
i_{n}+i \\
i
\end{array}\right)
$$

and they are part of number theory called multisection of series. Their properties have been developed in a series of beautiful papers by Carlitz $[3,4,5,6,7]$. They are related to lacunary recurrence formulas $[8,9]$ which were expounded by Lehmer [9], who developed them for the Bernoulli and Euler numbers [11].

\section{Multisections}

Multisection of series is a very old process which uses the primitive roots of unity to divide a given series into a number of sections [12]. If $r$ is a primitive $m$-th root of unity, that is $r^{m}=1$, $r \neq 1$, then the $k$-th $m$-section of the series $w(x)$ can be defined by 


$$
w(x ; m)=m^{-1} \sum_{j-1}^{m} w\left(r^{j} x\right) r^{m-k j}
$$

in which

$$
w(x)=w_{1}(x)=\sum_{n=0}^{\infty} w_{n} x^{n}
$$

is a generating function for Horadam's generalized sequence of numbers $\left\{w_{n}\right\} \equiv\left\{w_{n}(a, b ; p, q)\right\}$ [13], for which we define

$$
w_{n+1}(x)=\sum_{k=0}^{\infty} w_{n+k} \frac{x^{k}}{k !}(n=0,1,2, \ldots) .
$$

It follows that

$$
\begin{aligned}
w_{k}(x ; m)= & \frac{1}{m}\left(r^{m-k} w(r x)+r^{m-2 k} w\left(r^{2} x\right)+\ldots+r^{m-m k} w\left(r^{m} x\right)\right) \\
= & \frac{1}{m}\left(r^{m-k}\left(w_{0}+w_{1} r x+w_{2} r^{2} x^{2}+\ldots\right)+r^{m-2 k}\left(w_{0}+w_{1} r^{2} x+w_{2} r^{4} x^{2}+\ldots\right)\right) \\
& \quad+\ldots+r^{m-m k}\left(w_{0}+w_{1} r^{m} x+w_{2} r^{2 m} x^{2}+\ldots\right) \\
= & \frac{1}{m}\left(w_{0} \sum_{j=1}^{m} r^{m-j k}+w_{1} x \sum_{j=1}^{m} r^{m-j k+j}+\ldots+w_{k} x^{k} \sum_{j=1}^{m} r^{m-j k+j k}+\ldots\right) \\
= & \frac{1}{m}\left(w_{0} \frac{r^{m k}-1}{r^{k}-1}+w_{1} \frac{r^{m(k-1)}-1}{r^{k-1}-1}+\ldots+w_{k} x^{k} m r^{m}+\ldots\right) \\
= & w_{k} x^{k}+w_{k+2 m} x^{k+2 m}+\ldots
\end{aligned}
$$

Then with the corresponding Binet formula for the general terms:

$$
\begin{aligned}
\sum_{j=0}^{\infty} w_{k+j m} x^{k+j m} & =\sum_{j=0}^{\infty}\left(A(\alpha x)^{k+j m}+B(\beta x)^{k+j m}\right) \\
& =\frac{A \alpha^{k} x^{k}}{1-\alpha^{m} x^{m}}+\frac{B \beta^{k} x^{k}}{1-\beta^{m} x^{m}} \\
& =\frac{w_{k}-q^{m} w_{k-m} x^{m}}{1-v_{m} x^{m}+q^{m} x^{2 m}} x^{k}
\end{aligned}
$$

in which $v_{m}=\alpha^{m}+\beta^{m}$ is a Lucas primordial number expressed in terms of the roots, assumed distinct, of the characteristic equation of $\left\{w_{n}\right\}$. On equating coefficients of $x$ in (3.2), we can rearrange the terms to obtain the rather elegant result

$$
\frac{w_{k+m j}-v_{m} w_{k+m(j-1)}+q^{m} w_{k+m(j-2)}}{w_{k}-v_{m} w_{k-m}+q^{m} w_{k-2 m}}=\delta_{j, 0},
$$

where the right-hand side is the Kronecker delta. When $j$ is unity, we get Equation (3.16) of Horadam [14]. 


\section{Cycles of generalized binomial coefficients}

Carlitz [1] used the fact that

$$
\sum_{j=0}^{\infty}\left(\begin{array}{c}
i+j \\
j
\end{array}\right) v^{j}=\frac{1}{(1-v)^{i+1}} .
$$

A neat analogue of this can be readily developed from Carlitz and Riordan [15]: put $m=j, x=1$, $n=i, y=v$ in Equation (7.4) of that paper and

$$
\sum_{j=0}^{\infty}\left[\begin{array}{c}
i+j \\
j
\end{array}\right] v^{j}=\frac{1}{(v)_{i+1}},
$$

in which we have Carlitz' $q$-series analogue of the binomial coefficient $\left[\begin{array}{l}n \\ k\end{array}\right]=\frac{(q)_{n}}{(q)_{k}(q)_{n-k}}$, where [16]

$$
(q)_{n}=(1-q)\left(1-q^{2}\right) \ldots\left(1-q^{n}\right) .
$$

We also note a generalized cycle of Carlitz [17] which can be expressed as:

$$
\sum_{s=-m}^{m}(-1)^{s}\left[\begin{array}{l}
m+n \\
m+s
\end{array}\right]\left[\begin{array}{l}
n+p \\
n+s
\end{array}\right]\left[\begin{array}{c}
p+m \\
p+s
\end{array}\right] q^{\frac{1}{2} s(3 s+1)}=\frac{\underline{q}_{m+n+p} !}{\underline{q}_{m} ! \underline{q}_{n} ! \underline{q}_{p} !}
$$

in which there are Fermatian $q$-numbers [18] which can be expressed as

$$
\underline{q}_{n}=\left\{\begin{array}{cl}
-q^{n} \underline{q}_{-n} & n<0 \\
1+q+q^{2}+\ldots+q^{n-1} & n>0 \\
1 . & n=0 .
\end{array}\right.
$$

Here the $q$ is not necessarily the same as that of Horadam. For the origin of the more general $q$-series, see Eduard Heine [19].

We now investigate a cycle of length two with mixed coefficients:

$$
\begin{aligned}
\sum_{j=0}^{\infty} \sum_{k=0}^{\infty}\left[\begin{array}{c}
i+j \\
j
\end{array}\right]\left(\begin{array}{c}
j+k \\
k
\end{array}\right) v^{j} w^{k} & =\sum_{j=0}^{\infty}\left[\begin{array}{c}
i+j \\
j
\end{array}\right] \frac{v^{j}}{(1-w)^{j+1}} \\
& =\frac{1}{1-w} \sum_{j=0}^{\infty}\left[\begin{array}{c}
i+j \\
j
\end{array}\right]\left(\frac{v}{1-w}\right)^{j} \\
& =\left((1-w)\left(\frac{1-w}{v}\right)_{i+1}\right)^{-1} .
\end{aligned}
$$

These are developed further by extending ideas outlined and developed in Riordan [20] and Carlitz [1].

$$
\begin{aligned}
{\left[(1-v-w)-(1-w) u w^{-1}\right]^{-1} } & =\left((1-v-w)^{-1}\left[1-\frac{u(1-w)}{v(1-v-w)}\right]^{-1}\right) \\
& =\sum_{i=0}^{\infty} u^{i} w^{-i}(1-w)^{i}(1-v-w)^{-i-1} \\
& =\sum_{i, j, k=0}^{\infty}\left(\begin{array}{c}
i+j \\
j
\end{array}\right)\left(\begin{array}{c}
j+k \\
k
\end{array}\right) u^{i} v^{j} w^{k-i} .
\end{aligned}
$$


The coefficient of $w^{0}$ in (3.1) is the generating function

$$
\sum_{i, j=0}^{\infty}\left(\begin{array}{c}
i+j \\
j
\end{array}\right)\left(\begin{array}{c}
j+i \\
i
\end{array}\right) u^{i} v^{j}
$$

and the left-hand side of (3.1) can also be expressed as

$$
\begin{aligned}
{\left[1-v-w-(1-w) u w^{-1}\right]^{-1} } & =\left[1+u-v-\left(w+u w^{-1}\right)\right]^{-1} \\
& =\sum_{r=0}^{\infty}\left(w+u w^{-1}\right)^{r}(1+u-v)^{-r-1} \\
& =\sum_{r=0}^{\infty} \sum_{s=0}^{\infty}\left(\begin{array}{l}
r \\
s
\end{array}\right) \frac{u^{s} w^{r-2 s}}{(1+u-v)^{r+1}}
\end{aligned}
$$

and the coefficient of $w^{0}$ in this is

$$
\sum_{r=0}^{\infty}\left(\begin{array}{c}
2 r \\
r
\end{array}\right) \frac{u^{r}}{(1+u-v)^{2 r+1}}=\frac{1}{\sqrt{(1+u-v)^{2}-4 u}} .
$$

Thus,

$$
\begin{aligned}
\sum_{i, j=0}^{\infty}\left(\begin{array}{c}
i+j \\
j
\end{array}\right)^{2} u^{i} v^{j} & =\frac{1}{\sqrt{(1+u-v)^{2}-4 u}} \\
& =\frac{1}{\sqrt{(1-u-v)^{2}-4 u v}} \\
& =q\left(u v^{-1}, v\right),
\end{aligned}
$$

where

$$
q(x, y)=\sum_{n, m=0}^{\infty}\left(\begin{array}{c}
n+m \\
n
\end{array}\right) x^{n} y^{m}
$$

is an associated Legendre polynomial [21]. It is related to the Legendre polynomial when $n=m$. Some of these ideas lead naturally into problems associated with the Jackson calculus of sequences [22] and the Staudt-Clausen Theorem [23].

Henry Gould also pioneered some $q$-extensions of infinite sums of analogous sums [24] after proving similar results with ordinary binomial coefficients [25].

\section{Conclusion}

In this paper, we have made an attempt to find different patterns of non-zero distinct integer solutions to the bi-quadratic equation with three unknowns given by $11\left(x^{2}-y^{2}\right)+3(x+y)=10 z^{4}$. As bi-quadratic equations are rich in variety, one may search for integer solutions to other choices of bi-quadratic and higher order equations with multivariaties along with suitable properties. 


\section{References}

[1] Carlitz, L. (1965). Multiple sums and generating functions. Collectanea Mathematica. 17, 281-296.

[2] Riordan, J. (1958). An Introduction to Combinatorial Analysis. New York: Wiley, p.3.

[3] Carlitz, L. (1967). Rectangular arrays and plane partitions. Acta Arithmetica. 13, $29-47$.

[4] Carlitz, L. (1970). Some generalized Fibonacci identities. The Fibonacci Quarterly, 8, 249-254.

[5] Carlitz, L. (1965). Some multiple sums and binomial identities. SIAM Journal on Applied Mathematics, 13, 469-486.

[6] Carlitz, L. (1964). A binomial identity arising from a sorting problem. SIAM Review, 6, $20-30$.

[7] Carlitz, L. (1967). A binomial identity. SIAM Review, 229-231.

[8] Shannon, A. G. (1980). Some lacunary recurrence relations. The Fibonacci Quarterly. 18, 73-79.

[9] Aistleitner, C., Berkes , I., \& Tichy, R. (2010). Lacunary sequences and permutations. In I. Berkes, R. Bradley, M. Peligrad and R. Tichy. Dependence in Probability, Analysis and Number Theory: A Volume in Memory of Walter Philipp. Heber City, UT: Kendrick Press, $35-49$.

[10] Lehmer, D. H. (1935). Lacunary recurrence formulas for the numbers of Bernoulli and Euler. Annals of Mathematics, 36, 637-649.

[11] Benjamin, A. T., Chen, B., \& Kindred, K. (2010). Sums of evenly spaced binomial coefficients. Mathematics Magazine, 83, 310-373.

[12] Beauregard, R. A., \& Dobrushkin, V. A. (2016). Multisection of series. The Mathematical Gazette. 100, 549, 460-470.

[13] Horadam, A. F. (1965). Generating functions for powers of a certain generalized sequence of numbers. Duke Mathematical Journal, 32, 437-446.

[14] Horadam, A. F. (1965). Basic properties of a certain generalized sequence of numbers. The Fibonacci Quarterly, 3, 161-176.

[15] Carlitz, L., \& Riordan, J. (1964). Two element lattice permutation numbers and their $q$-generalization. Duke Mathematical Journal, 31, 317-388.

[16] Carlitz, L. (1962). Note on a $q$-identity. Univerzitet u Beogradu. Publikacije Elektrotehničkeg Fakulteta. Serija Matematika i Fizika, 78-83, 19-20.

[17] Carlitz, L. (1955). Note on a q-identity. Mathematica Scandinavica, 3, 281-282.

[18] Shannon, A. G. (2014). Fermatian Hurwitz Series within the Fontené-Jackson Calculus. Advanced Studies in Contemporary Mathematics, 24 (1), 129-136. 
[19] Heine, E. 1847. Untersuchungen über die Reihe $1+\ldots x^{2}+\ldots$. Journal für die Reine und Angewandte Mathematik, 34, 285-328.

[20] Riordan, J. (1968). Combinatorial Identities. New York: Wiley, pp. 45, 202.

[21] Carlitz, L. (1954). Note on Legendre polynomials. Bulletin of the Calcutta Mathematical Society, 46, 93-95.

[22] Shannon, A. G. (2003). Generalized Bernoulli Polynomials and Jackson's Calculus of Sequences. Notes on Number Theory and Discrete Mathematics, 9 (1), 1-6.

[23] Horadam, A. F., \& Shannon, A. G. (1976). Ward's Staudt-Clausen problem. Mathematica Scandinavica, 29, 239-250.

[24] Gould, H. W. (1961). The $q$-series generalization of a formula of Sparre Andersen. Mathematica Scandinavica, 9, 90-94.

[25] Gould, H. W. (1958). Note on a paper of Sparre Andersen. Mathematica Scandinavica, 6, $226-230$. 